\title{
Analysis of Shockwave Front-Time Characteristics based On Pulse Discharge in Water
}

\author{
Dong Yan ${ }^{1}$, Qiong $\mathrm{Wu}^{1{ }^{1 *}}$, Inchen Chen ${ }^{2}$ and Nan $\mathrm{Zhao}^{2}$ \\ ${ }^{1}$ College of Architecture and Civil Engineering, Xinyang Normal University, Xinyang 464000, China \\ ${ }^{2}$ College of International Exchange, Krirk University, Bangkok 10220, Thailand
}

Received 30 March 2020; Accepted 26 July 2020

\begin{abstract}
Strong shockwaves can be formed by pulse discharge in water, and are applicable in fields such as control blasting, oil production, and national defence construction. Wave front-time is an important parameter in shockwave theory and effect. In order to reveal the relationship between the wave front-time and electrical energy and hydrostatic pressure, this study proposed a wave front-time analysis model. The relations between these conditions and shockwave front-time were derived and combined with theoretical information. The shockwave front-time was obtained at several sites under different conditions of hydrostatic pressure, breakdown energy, and propagation distance. Results indicate that shockwave front-time decreases with increasing breakdown energy, when electrical energies are in the range of 1500$3500 \mathrm{~J}$. Wave front-time increases with increasing hydrostatic pressure, when hydrostatic pressures are in the $0-4 \mathrm{MPa}$ range. Wave front-time increases with increasing propagation distance within $4 \mathrm{~m}$. The breakdown energy and hydrostatic pressure have significant effects on the increase of the front-time as the propagation distance increases. The findings provide references for experiments on pulse discharge in water and evaluation of shockwave performance.
\end{abstract}

Keywords: Pulse discharge in water, Shockwave front-time, Breakdown energy, Hydrostatic pressure, Propagation distance

\section{Introduction}

Since the invention of underwater pulse discharge technology, scholars have studied its application in industrial manufacturing. With technological progress and improvement of equipment, the technology of pulse discharge in water has been gradually accepted in scientific and industrial communities. Electrical energy produces a strong impact that can cause mechanical damage by utilizing shockwaves. Electrohydraulic effect has been widely applied in many fields and is promising in rock-mass fracturing. Compared with traditional rock-mass fracturing methods that use explosives, the pulse discharge method is characterized by controllable energy, high precision, high repeatability, safety, and reliability. Therefore, the pulsed discharge in water has attracted attention in the rock-mass fracturing sector.

Shockwave front-time is the time for the local pressure to reach its peak value after the shockwave front arrives at a given position [1]. The front-time reflects the action time of the shockwave at a certain position, and is an important parameter in analyzing the effect of a shockwave [2]. For shockwaves acting on a rock, the front-time mainly reflects the loading speed of shockwave pressure, which is the main feature of shockwave energy release. The shockwave produces a continuous breaking effect on the rock in the front-time and is an important factor that forms rock fracture areas [3]. Duration (front-time) reaches a certain value, and the effect is mainly reflected in fracture and plastic areas, thereby effectively extending the fracture of rocks. If the front-time is extremely short, then the shockwave energy effects are concentrated in the surface region, and a small

*E-mail address: wugiong180216@163.com

ISSN: 1791-2377 @ 2020 School of Science, IHU. All rights reserved. doi:10.25103/jestr.134.03 amount of energy is transferred for internal fractures. Conversely, a longer front-time tends to promote the formation of internal fractures. However, if the front-time is extremely long, then the energy release becomes decentralized, which is disadvantageous to achieve a good shock effect [4]. Therefore, shockwave front-time is a crucial subject for scientific study, industrial production, and national defense.

Based on this concept, scholars have conducted numerous studies on shockwaves of underwater pulse discharge, whereas few studies have been performed on the wave front rising time of shockwaves [5-13]. Thus, a key problem is how to accurately obtain the wave front-time characteristics of shockwave and determine the relationship between electrical energy and water pressure.

Therefore, a combination of theoretical and experimental research is adopted in this study to analyze the characteristics of shockwave rising time, discuss the action characteristics of wave front-time under different electrical energy and water pressure, and establish the model of wave front-time to provide the basis for the optimization of pulse discharge technology in water.

\section{State of the art}

A number of studies have been conducted on the phenomenon of shockwave generated by pulse discharge in water. Godwin [5] mainly analyzed the dynamic characteristics of plasma bubbles and studied the acoustic characteristics of shockwaves generated during expansion. Gurovich [6] theoretically analyzed the resistance of the plasma channel of pulsed discharge in water in the experiment, and approximately discussed the time-varying model of arc resistance to obtain a preliminary 
understanding of the arc resistance problem. Eubank [7] proposed that the pressure and temperature in the arc could be approximately considered to be homogeneous. Bruggeman [8] analyzed the breakdown properties of water under high-voltage pulse discharge, and the results obtained were close to the actual situation. In the underwater shockwave theory proposed by Touya [9], the empirical formula of peak pressure of the shockwave was presented; the study showed that the breakdown process could be realized only if the energy applied to the deposition in the gap could reach $200 \mathrm{~J}$. Yang [10] also found the phenomenon of plasma energy transfer in the experiment, which explained the formation of shockwave from another aspect. Kochetkov [11] proposed the theory of shockwave superposition under limited water fields. High hydrostatic pressure was helpful in stabilizing shockwave propagation and effectively improving the influence range of the shockwave. In the shockwave focusing experiment on underwater pulse discharge, Zhang Zhenfu [12] obtained the reflection characteristics of shockwaves. Through experimental studies, Yin Zhiqiang [13] summarized the pressure characteristics of shockwaves and the voltage and current characteristics during the breakdown process and analyzed the influence of voltage and water pressure on the generation of shockwaves. Yan Dong [14,15] experimentally investigated the characteristics of shockwave pressure in underwater pulse discharge and formulated the attenuation law of shockwave pressure. Lu Xinpei [16] analyzed the characteristics of a plasma channel of pulsed discharge in water and the bubble breakage process. Qiu Aici [17] performed a detailed experiment on the breakdown of highvoltage pulse discharge in water and analyzed the breakdown characteristics of the 100-ns pulse. Sun Yaohong [18] focused on applications of underwater pulse discharge technology and determined the electrotechnical characteristics and shockwave pressure characteristics of underwater pulse discharge.

The aforementioned studies mainly focused on the pressure characteristics of shockwaves generated by pulse discharge in water, but few studies have been conducted on the wave front rising properties. This study adopts a method that combines theory and experiment and establishes a model of the wave front-time. Furthermore, the action characteristics of wave front-time under different electrical energy and water pressure are discussed and the wave fronttime relationship is formed so that the basis for the optimization of pulse discharge technology in water can be provided.

The rest of this study is organized as follows. Section 3 describes the specific experimental methods and constructs the wave front-time analysis model of impact wave. In section 4 , the wave front-time characteristics of the model are analyzed through the experimental data, and the effect of electrical energy and water pressure on the wave front-time is determined. Section 5 summarizes relevant conclusions.

\section{Methodology}

\subsection{Shockwave front-time model}

When a shockwave is generated, irregular fluctuation usually occurs at the initiating point mainly due to shortterm oscillatory interference caused by reflection, which causes difficulty in accurately determining the shockwave starting position and front-time. Therefore, a front-time algorithm was proposed to effectively reduce statistical error caused by this irregular fluctuation.

Front-time $(T)$ is a physical parameter that can be directly observed from the waveform images. Figure 1 shows a typical measured pressure-time curve of a shockwave, related to $t_{l}$, the time for the shockwave pressure to increase from $10 \%$ to $90 \%$ of peak pressure, where A marks $10 \%$ peak pressure $\left(0.1 P_{1}\right)$ and B marks $90 \%$ peak pressure $\left(0.9 P_{1}\right) . P_{l}$ is shockwave peak pressure. We define front-time as $T=1.25 t_{1}[19]$.

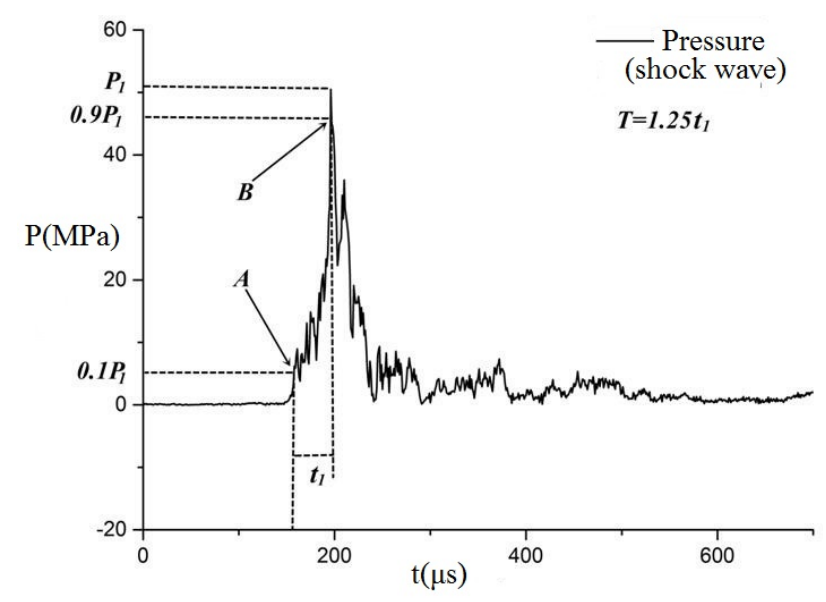

Fig. 1. Typical measured shockwave pressure

Figure 2 shows the piston model of shockwave front, and Figure 3 shows the corresponding waveform at the measurement position [20]. In the figures, $\rho_{0}, P_{0}, \rho_{1}$, and $P_{1}$ represent the density and pressure of water before and after the wave front, respectively; $t_{0}$ is the shockwave arrival time at position $x_{0} ; \mathrm{T}$ is the front-time, as discussed above; $t_{0}+T$ is the time for the pressure at $x_{0}$ to reach peak value, $P_{1}$, at which time the wave front reaches $x_{1} ; \Delta x$ is the thickness of the shockwave; and $U_{s}$ is the shockwave propagation velocity (approximately $1500 \mathrm{~m} / \mathrm{s}$ in water). The wave front is extremely thin, so flow within the wave front can be considered as quasi-steady, and $U_{s}$ is constant [21]. The density of water changes slightly with hydrostatic pressure; thus, we can assume that $\rho_{0}=\rho_{1}$.

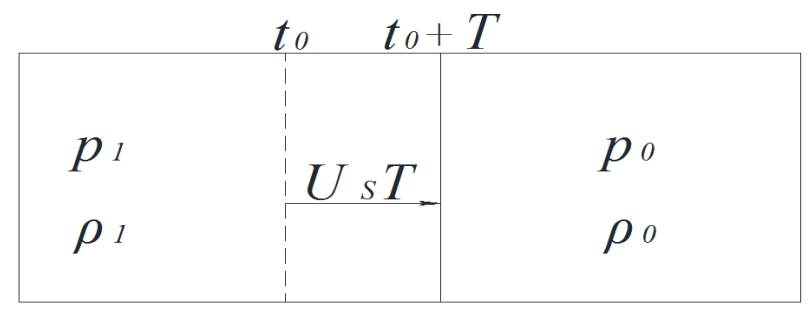

Fig. 2. Piston model of shockwave front

Following the shockwave model in Figures 2 and 3, we can determine the relationship between front-time (T) and shockwave thickness $(\Delta x)$ as follows:

$T=\frac{\Delta x}{U_{s}}=\frac{P_{0} l}{\left(P_{1}-P_{0}\right) U_{s}}$ 


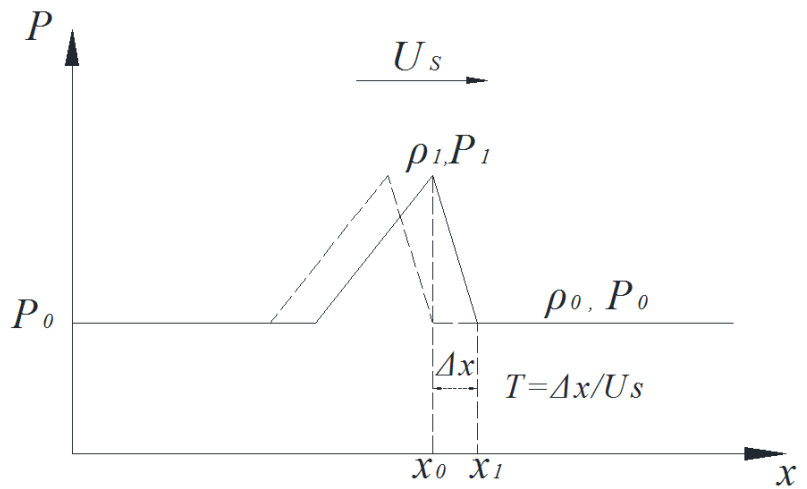

Fig. 3. Waveform diagram of shockwave front arriving at measurement particle

Where $\Delta x$ is the thickness of the shockwave, $P_{1}$ is the peak pressure of the shockwave at the measurement position, and $l$ is the mean free path of a water molecule [22]. The discharge electrode gap used to create the shockwave is extremely small $(5 \mathrm{~mm})$ and may be considered as a point source. Shockwave intensity undergoes exponential decay with increasing distance [23]. Thus, the peak shockwave pressure is

$P_{1}=P_{m} e^{-\frac{\gamma d}{U_{s}}}$

Where $P_{m}$ is the shockwave peak pressure in the discharge breakdown channel, $\gamma$ is the exponential decay constant, and $\mathrm{d}$ is the distance from the discharge channel. Previous studies [24, 25], proposed the following accurate method to calculate $P_{m}$ :

$P_{m}=\eta \sqrt{\frac{\rho_{0} W_{r}}{\tau t}}$

Where $\eta$ is a non-dimensional complex integral function with an approximate value of 0.75 for water, $\rho_{0}$ is the density of water, $\tau$ is the time for the ascent stage of the discharge current waveform (as shown in Figure 6, $\tau$ is the time from $t_{b}$ to the discharge current peak), $t$ is the duration of release of pulse energy [26], and $W_{r}$ is the pulse energy of the discharge channel in a unit length, i.e., $W_{r}=W / r$, where

$W$ is the discharge breakdown energy in water and $r$ is the electrode spacing, i.e., $5 \mathrm{~mm}$. These values can be obtained through experiment.

The peak shockwave pressure at any measurement point can be obtained by combining equations (2) and (3) as follows:

$$
P_{1}=\eta \sqrt{\frac{\rho_{0} W}{r \tau t}} e^{-\frac{\gamma d}{U_{s}}}
$$

and substituting equation (4) into (1), the approximate expression for front-time is

$$
T=\frac{P_{0} l}{\left(\eta \sqrt{\frac{\rho_{0} W}{r \tau t}} e^{-\frac{\gamma d}{U_{S}}}-P_{0}\right) U_{s}}
$$

\subsection{Fundamental physical process}

During the breakdown of a water dielectric by a high-voltage pulse discharge, the liquid phase-gas phase-plasma transformation is finished within a very short time, forming a high-temperature, high-pressure plasma channel. The huge pressure gradient in the channel due to the high pressure interior and temperature gradient at the plasma boundary leads to rapid outward expansion of the plasma channel, which achieves a high-speed transformation from electrical to mechanical energy [27, 28]. Mechanical energy is rapidly released in water in the form of a shockwave. The outward expansion of plasma from the discharge channel forms the shockwave, and to a certain extent the peak pressure rise time is dependent on the expansion velocity of the plasma channel, with faster expansion velocity producing a shorter rise time. This rise time is called shockwave front-time, which reflects the effective time of mechanical energy acting on a particle.

In this period, the physical properties of the particle change significantly, and it is the main stage that causes a strong shock. The front-time $(T)$ is dependent on a number of parameters, such as breakdown energy $(W)$, propagation distance $(d)$, and hydrostatic pressure $\left(P_{0}\right)$. The shockwave is the main carrier of discharge energy in water, and the front-time of the shockwave is directly affected by the breakdown energy. Shockwave energy decays in propagation, and so the front-time is different for various propagation distances. Hydrostatic pressure restrains the discharge breakdown and plasma expansion, and the shockwave is damped with the propagation process in water, also producing different shockwave front-times with this process.

\subsection{Experimental equipment}

The experimental equipment consisted of the experimental device and the system for measuring the pulse discharges in water. The experimental device for measuring pulse discharges in water is shown schematically in Figure 4. The pulse power supply provided DC at high voltage in the 6-15 $\mathrm{KV}$ range. The rated capacitance was $60 \mu \mathrm{F}$, and the energy storage limit was $7000 \mathrm{~J}$. The electrodes were made of coaxial steel tube and copper bar. A schematic and photograph of the electrode structure are shown in Figure 5. The space between the positive and negative electrodes was $5 \mathrm{~mm}$. The electrodes were located at the end of a tube with an inner diameter of $100 \mathrm{~mm}$ and a length of $4000 \mathrm{~mm}$. Five sensor connectors (Nos. 1-5) were uniformly distributed on one side of the tube wall, $500 \mathrm{~mm}$ apart. The distance from the electrode to the nearest (No. 1) sensor connector was $1000 \mathrm{~mm}$. The tube was filled with tap water with a conductivity of approximately $1.3 \mathrm{~S} / \mathrm{m}$. The pre-set hydrostatic pressure was adjusted by an external hydraulic pump with a maximum capacity of $12 \mathrm{MPa}$.

The measuring system in this study was composed of six parts: a Rogowski coil, a P6015A high-voltage probe, a DSO6014A oscilloscope, piezoresistive pressure sensors, a transient recorder, and a computer. The sensitivity of the Rogowski coil was $38 \mathrm{KA} / \mathrm{V}$, which was suitable for measuring the pulse current due to its high measurement precision [29]. The P6015A high-voltage probe could meet 
the acquisition accuracy for transient voltage [30]: the maximum input voltage was $20 \mathrm{kV}$, the rise time was $4.0 \mathrm{~ns}$, and the compensation range was $7-49 \mathrm{pF}$. The sampling rate of the DSO6014A oscilloscope was $4 \mathrm{GSa} / \mathrm{a}$. The CY200 piezoresistive pressure sensor had a measuring range of 50 $\mathrm{MPa}$, a natural frequency of $1 \mathrm{MHz}$, and a comprehensive accuracy of $0.1 \%$ FS. For the TST6250 transient recorder, the maximum sampling rate was $20 \mathrm{M}$ sps, the bandwidth was $1 \mathrm{MHz}$, and the comprehensive precision was $0.1 \% \mathrm{FS}$. The test system used in this study had advantages such as simple corollary equipment, high sampling frequency, and good interference resistance. Independent DC power supplies were used to avoid conduction coupling interference caused by the high-voltage discharge process [31].

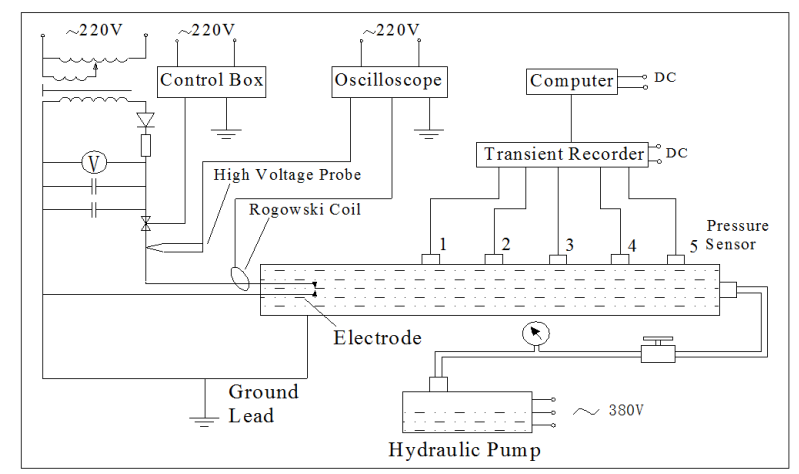

Fig. 4. Schematic of experimental device

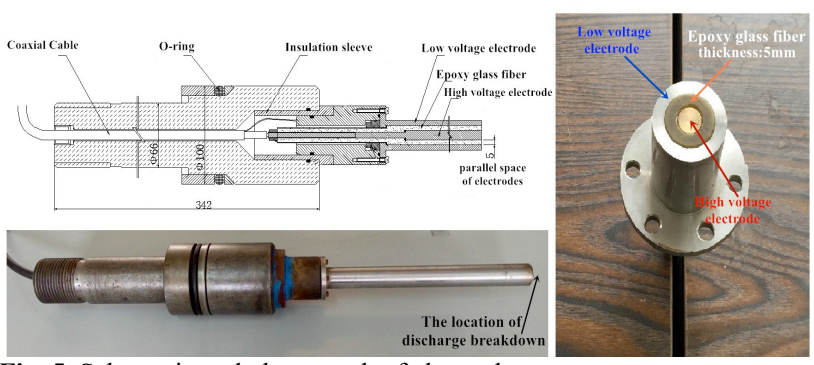

Fig. 5. Schematic and photograph of electrode structure

\subsection{Experimental scheme}

Shockwave pressures were measured at five sensor locations to investigate the influence of discharge energy, hydrostatic pressure, and propagation distance on the shockwave fronttime. The tube was filled with water and hydrostatic pressure was supplied by an external water pump. The equipment could withstand $12 \mathrm{MPa}$. Hydrostatic pressure was applied in five gradients: $0,1,2,3$, and $4 \mathrm{MPa}$. Breakdown energy was derived from the breakdown voltage current, measured by a high-precision current testing coil and high voltage probe. Five breakdown energies were investigated: 1500, 2000, 2500, 3000, and $3500 \mathrm{~J}$. Thus, 25 independent experiments were conducted incorporating all of the hydrostatic pressures and breakdown energies, with five discharge tests for each experiment, and the pressure waveform of the first shockwave was measured at different points. Only the first shockwave was considered because of the reflection from the bottom of the tube, and the reflection wave was not studied.

\subsection{Discharge breakdown measurement}

During the breakdown of the water dielectric by electrode discharge, a large amount of energy was released within a short time. The breakdown energy $(W)$ was obtained by measuring the transient current and voltage. The breakdown energy was $3000 \mathrm{~J}$, and the hydrostatic pressure was $1 \mathrm{MPa}$. The yellow curve in Figure 6 represents the current function $I(t)$, and the green curve in Figure 7 represents the voltage function $U(t)$. In addition, the maximum charging voltage $\left(U_{m}\right)$, breakdown voltage $\left(U_{b}\right)$, and breakdown time $\left(t_{b}\right)$ are also marked.

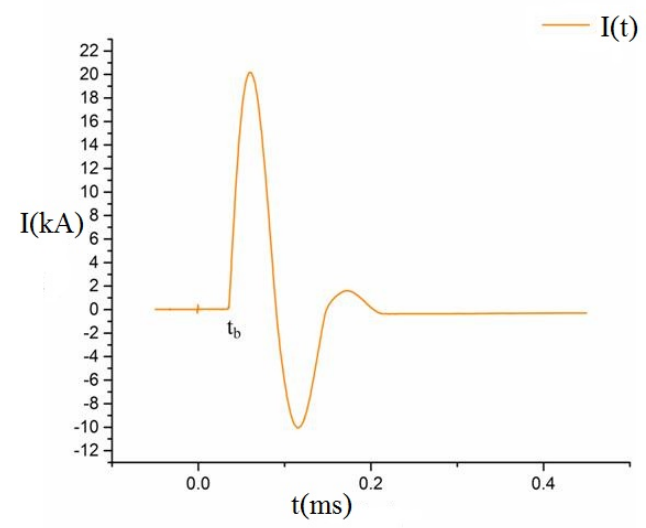

Fig. 6. Oscillogram of discharge current $I(t)$

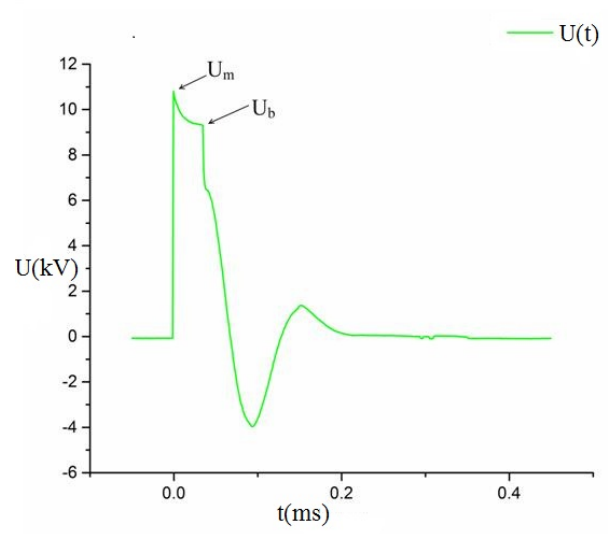

Fig. 7. Oscillogram of discharge voltage $U(t)$

The discharge process can be described as follows: the discharge starts when the capacitor is charged to the rated voltage. The maximum charging voltage decreases slowly before breakdown of the water dielectric due to the influence of conductivity. The voltage decreases greatly at the moment of breakdown of the water dielectric. Then the voltage decreases as the current increases, and electrical energy is rapidly released into the breakdown channel. The breakdown voltage is the transient voltage at the moment of breakdown of the water dielectric. This time is referred to as the breakdown time where the moment of discharge is time 0 . Among the high-voltage pulse discharge characteristics, the breakdown voltage is the major factor that determines the breakdown energy, and the breakdown energy directly influences shockwave formation.

\section{Result Analysis and Discussion}

\subsection{Effect of breakdown energy}

Figure 8 shows the breakdown energy and front-time of sensor 1 at a fixed distance to the discharge electrode under different hydrostatic pressures. 


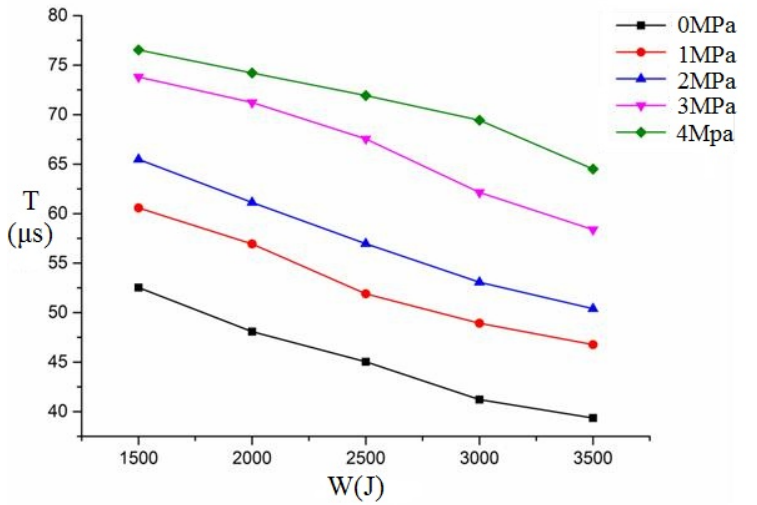

Fig. 8. Breakdown energy $(W)$ and front-time $(T)$ at sensor 1

Front-time decreases with increasing breakdown energy, reducing by approximately $3.9 \mu$ shen the breakdown energy increases by $500 \mathrm{~J}$. For the breakdown energy in the experimental range, the measured front-time is at the microsecond level (with distribution of approximately 30-90 $\mu \mathrm{s})$. Front-time reduction was approximately equal under different hydrostatic pressure conditions, i.e., the curve slopes in Fig. 8 are similar. This condition indicates that the front-time may be effectively controlled by breakdown energy, i.e., breakdown energy is the major influencing factor in the formation of shockwave front-time.

Front-time decreases slightly as breakdown energy increases for different hydrostatic pressures. The main reason could be that higher discharge energy requires higher potential energy at the electrodes, which was advantageous to rapid formation of the plasma channel as well as energy release, i.e., higher discharge energy produced larger power density in the plasma channel. Furthermore, faster plasma expansion velocity shortens the shockwave formation time. Consequently, the front-time was reduced. The breakdown energy showed an approximately inverse proportional relationship with front-time.

\subsection{Effect of hydrostatic pressure}

Figure 9 shows the hydrostatic pressure and front-time under different breakdown energy conditions for sensor 1. Fronttime increases with rising hydrostatic pressure, and this trend is stable under different breakdown energy conditions. Front-time increased by approximately $3.3 \mu$ s on average for every $1 \mathrm{MPa}$ increase. Hydrostatic pressure shows an approximately linear relationship with the front-time.

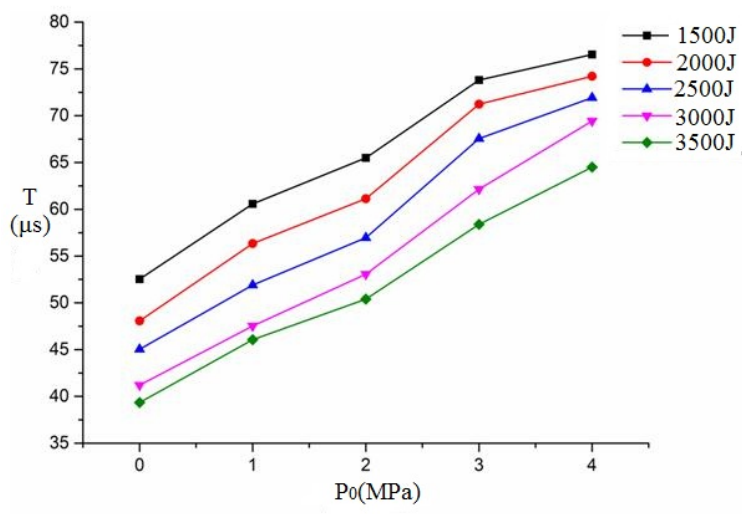

Fig. 9. Hydrostatic pressure $\left(P_{0}\right)$ and front-time $(T)$ for sensor 1
The approximately linearly increasing relationship of $P_{0}-T$ can be attributed to two reasons. First is plasma formation speed in the discharge breakdown. Increased hydrostatic pressure produced a slight increase in breakdown channel resistance, which hindered the plasma channel formation and reduced the plasma formation speed. The time to form the shockwave was prolonged, and the front-time increased accordingly. Second, water density and hydrostatic pressure resistance affected plasma expansion [32]. The plasma channel was influenced by water density and hydrostatic pressure resistance. Hydrostatic pressure was controlled at $0-4 \mathrm{MPa}$, and water may be considered incompressible; thus, the change in the water density over this range was negligible [33]. Only hydrostatic pressure affected plasma expansion, with higher hydrostatic pressure slowing plasma expansion velocity and increasing front-time.

\subsection{Effect of propagation distance}

Front-time differences with increasing propagation distance mainly arise from two factors. One is water medium factor. Shockwaves exponentially decay with the propagation process due to water damping, with decreased energy producing an increasing front-time. The other factor is the unloading wave of bubble pulsation factor. Furthermore, during the evanescent bubble expansion and contraction, the rarefaction wave from the bubble surface has an unloading effect on the shockwave tail. However, bubble expansion velocity is extremely slow, and has minimal influence on shockwave energy decay. Therefore, this effect was not considered in the present study. Consequently, the influence of propagation distance on front-time was through the breakdown energy and hydrostatic pressure.

First, the effect of breakdown energy on shockwave front-time with propagation was investigated with fixed hydrostatic pressure. Sensors 1-5 were used to measure front-time with different propagation distances, as shown in Figure 10. Hydrostatic pressure was $1 \mathrm{MPa}$, and breakdown energy varied in the range of 1500-3500 J.

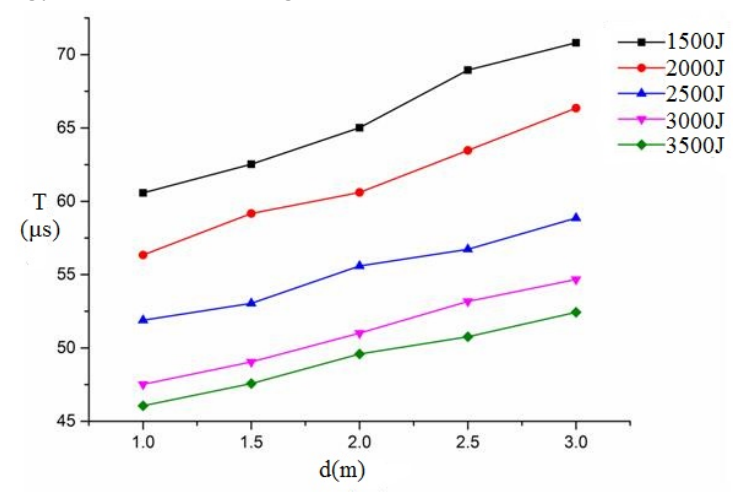

Fig. 10. Effect of breakdown energy on front-time propagation

Front-time increases with increasing propagation distance, and the $d-T$ slope of the curve decreases gradually with increasing breakdown energy. Increasing the propagation distance reduces the shockwave energy, which reduces the rise speed of the shockwave peak pressure; thus, the front-time increases. Therefore, increasing the propagation distance slightly increases the front-time.

Higher breakdown energy results in less shockwave attenuation rate with propagation. Consequently, the fronttime slows and the slope decreases due to the slowness of the shockwave energy attenuation, e.g., the slope of breakdown energy $W=3500 \mathrm{~J}$ is significantly less than that of $W=1500 \mathrm{~J}$. Therefore, the energy considerably influences the 
shockwave propagation. Shockwave energy can compensate for the energy loss of the wave head, so higher shockwave energy can effectively maintain the waveform in propagation. When the breakdown energy gradually increases to a certain value, the increasing slope of the front-time due to the energy factor gradually tends to be stable. However, the front-time difference due to the increasing $W$ gradually decreases, e.g., the front-time difference between the $1500 \mathrm{~J}$ and $2000 \mathrm{~J}$ cases is approximately $4.5 \mu \mathrm{s}$, whereas it is only $1.6 \mu$ s between the $3000 \mathrm{~J}$ and $3500 \mathrm{~J}$ cases.

Hydrostatic pressure significantly affected the front-time with the propagation process, as shown in Fig. 11, for the breakdown energy $W=2500 \mathrm{~J}$ and hydrostatic pressure 0 $4 \mathrm{MPa}$, using sensors $1-5$ to measure the front-times at different propagation distances. Higher hydrostatic pressures produce lower $d-T$ slopes, i.e., higher hydrostatic pressures have an effect on slower growth of the front-time with propagation.

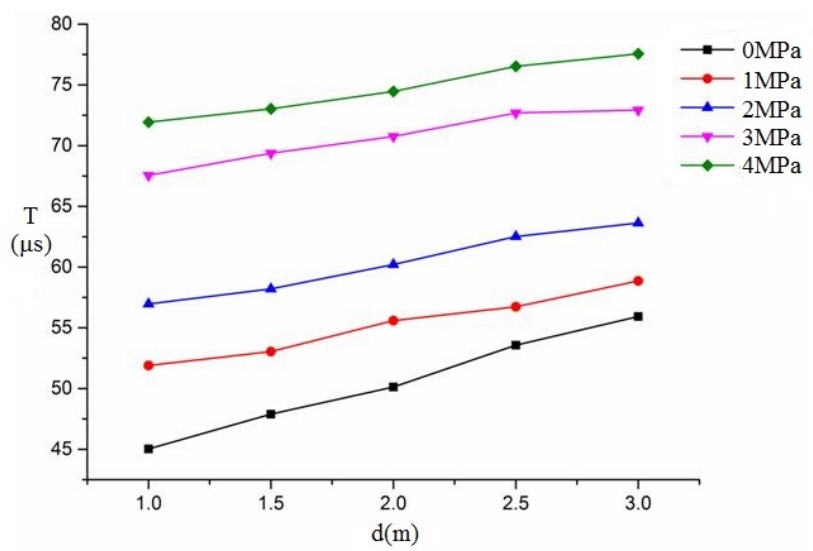

Fig. 11. Effect of hydrostatic pressure on front-time propagation

The main reasons for the decreased $d-T$ slopes with increased hydrostatic pressures are shockwave attenuation with propagation at different hydrostatic pressure mediums. Increased hydrostatic pressure enhances the shockwave energy transfer, thereby reducing the energy loss and shockwave attenuation. Consequently, the front-time slowly increases because of the energy efficient transfer of the shockwave. The stable transfer of the shockwave energy plays an important role in the steady increase of the fronttime.

The front-time has an irregular large jump when hydrostatic pressure varies from $2 \mathrm{MPa}$ to $3 \mathrm{MPa}$, while it increases regularly for the other ranges. This condition is due to an environmental mutation in the high voltage pulse discharge breakdown process. Many small bubbles are produced during water injection into the equipment. When the hydrostatic pressure exceeded a certain value (approximately in $3 \mathrm{MPa}$ range for the present study), the small bubbles collapsed and disappeared. The water medium was not easy to break down when small bubbles disappeared, thereby increasing the breakdown time at discharge. Compared with the hydrostatic pressure of 0-2 $\mathrm{MPa}$, when the hydrostatic pressure reaches $3 \mathrm{MPa}$, the bubbles in water disappear, leading to the mutation of breakdown environment. As a result, the breakdown time increases further, which directly influences the shockwave rise time to peak pressure, and consequently increases the front-time more than the effect expected from the increased hydrostatic pressure.

\section{Conclusions}

To explore the characteristics of the shockwave based on the pulse discharge in water, and to reveal the relationship between the rising time of shockwave pressure, energy, and hydrostatic pressure, we adopted a method that combines experimental and theoretical study to analyze the wave front-time characteristics of the shockwave. The following conclusions could be drawn:

1) The shockwave front-time decreases with increasing breakdown energy. The rate of the front-time decrease is highly similar under different hydrostatic pressure conditions, and the influence of breakdown energy on the front-time is relatively stable. Higher breakdown energy produces faster plasma expansion velocity, and thus shorter shockwave front-time.

2) The shockwave front-time increases with increasing hydrostatic pressure. The increased hydrostatic pressure hinders the discharge breakdown and plasma expansion processes. Consequently, the shockwave rise time to peak pressure is slowed, and the front-time is prolonged.

3) The shockwave front-time increases with the increasing propagation distance. The shockwave energy attenuation with propagation has a negative effect on the rise speed to peak pressure and increases the front-time.

4) The increasing front-time with propagation is slower for higher breakdown energy than a lower one. This condition shows that the shockwave energy can compensate for energy loss at the wave head. Thus, the higher energy can effectively maintain the waveform in the propagation process and maintain the front-time steady growth.

5) Increasing the front-time with the propagation process is slower for the higher hydrostatic pressure. The increased hydrostatic pressure is advantageous to the shockwave energy transfer, thereby reducing energy loss in the propagation process, and ensuring that the attenuation of the shockwave is stable. Consequently, the front-time slowly increases with the shockwave energy efficient transfer.

Thus, a new understanding of the wave-front rising time of the shockwave was proposed in this study. Analyzing the characteristics of the shockwave is relevant to the development of pulse discharge technology in water. Owing to the limited number of holes in the pipeline experimental table, the data collected from the experiments are also limited in the future and a porous pipeline experimental table can be applied. Additional influencing factors should be considered in future studies to improve our understanding of the behavior of the shockwave front-time.

\section{Acknowledgements}

This work was supported by the Nanhu Scholars Program for Young Scholars of Xinyang Normal University.

This is an Open Access article distributed under the terms of the Creative Commons Attribution License

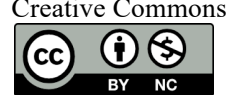




\section{References}

1. Pain H. J., Chen N. X., Hao S. G., "The Physics of Vibrations and Waves". Beijing: People's Education Press, China, 1980, pp. 2-6.

2. Tang W. H., "Shock Wave Physics". Beijing: Science press, China, 2011, pp. 13-15.

3. Cho. S. H., Katsuhiko K., "Influence of the applied pressure waveform on the dynamic fracture processes in rock". International Journal of Rock Mechanics and Mining Sciences, 72(41), 2004, pp. $771-784$

4. Chen J. X., "Analysis of Correlation Factors Between Stress Wave and Rock Fracture". Chinese Journal of Rock Mechanics and Engineering, 16(2), 1997, pp.148-154.

5. Godwin R. P., Chapyak E. J., "Recource letter BD-1:Bubble dynamics". American Journal of Physics, 63(1), 2000, pp. 211-222.

6. Gurovich V. T., Grinenko A., "Simplified model of underwater electrical discharge". Physical Review E, 69(1), 2004,036402.

7. Eubank P. T., Patel M. R., Barrufet M. A., "Theoretical models of the electrical discharge machining processes.III. The variable mass, cylindrical plasma model”. Journal of Applied Physics, 73(11), 1993, pp. 7900-7909.

8. Bruggeman P., Leys C., "Non-thermal plasmas in and in contact with liquids". Journal of Physics D: Applied Physics, 42(5), 2009, 053001 .

9. Touya G., Reess T., Pecastaing L., "Development of subsonic electrical discharges in water and measurements of the associated pressure waves". Journal of Physics D: Applied Physics, 39(24), 2006, pp. 5236-5244.

10. Yang B., Lei L. C., Zhou M. H., "Effects of the Liquid Conductivity on Pulsed High-voltage Discharge Modes in Water". Chinese Chemical Letters, 15(10), 2004, pp.1215-1218.

11. Kochetkov, Pinaev A. V., "Shock wave processes in wire explosion in water and bubbly media". Combustion Explosion And Shock Waves, 51(6), 2015, pp.722-731.

12. Zhang Z. F., Zeng X. W., Wang Y. B., "Experimental Study of the focusing property of underwater pulsed discharge shock wave". Journal of National University of Defense Technology, 34(4), 2012, pp.54-57.

13. Yin Z. Q., Zhao J. C., Bian D. C., "Research on the Delay Characteristics and Current Characteristics of High-voltage Pulse Discharge in Water". Journal of Taiyuan University of Technology, 47(3), 2016, pp.326-336.

14. Yan D., Bian D. C., Zhao J. C., Niu S. Q., "Study of the electrical characteristics, shock-wave pressure characteristics and attenuation law based on pulse discharge in water". Shock and Vibration, 2016, 6412309.

15. Yan D., Zhao J. C., Niu S. Q., "Normal reflection characteristics of one-dimensional unsteady flow shock waves on rigid walls from pulse discharge in water". Shock and Vibration, 2017, 6958085.
16. Lu X. P., Pan Y., Zhang H. H., "A study on the characteristic of plasma and bubble break process of pulsed discharge in water". Chinese Journal of Physics, 51(8), 2002, pp. 1768-1769.

17. Jia W., Qiu A. C., Sun F. J., "Effects of the Pressure Under the Several Hundred Nanosecond Pulse on the Breakdown Characteristics of the Water Switch". High Voltage Engineering, 32(1), 2006, pp. 50-52.

18. Sun Y. H., Zuo G. N., "Experimental Study of the Electrical Parameters of the Transmission Spark Source". High Voltage Engineering, 27(3), 2001, pp.39-41.

19. Jia S. H., Zhao J. C., Yin Z. Q., Bian D. C., Yan D., "Research on change laws of front-time in water shock wave based on pulsed high voltage discharge in permeability enhancement in coal seams". Journal of Taiyuan University of Technology, 46(6), 2015, pp.680684.

20. Yao X. L., Wang Y., Zhang A. M., "Underwater explosion bubble dynamics". Harbin: Harbin Engineering University Press, China, 2011, pp.12-19.

21. Li W. X., "One-Dimensional Nonsteady Flow and Shock Waves". Beijing: National Defence Industry Press, China, 2003,pp.131-138.

22. White F. M., "Fluid Mechanics". New York: The McGraw-Hill Companies Inc, USA, 2011, pp.95-104.

23. Sun B., "Discharge Plasma in Liquid and Its Applications". Beijing: Science press, China,2013, pp.72-79.

24. Qin Z. Y., Zuo G. N., "High-voltage Strong-pulse Discharge and Its Applications". Beijing: Beijing University of Technology Press, China, 2000, pp.135-142.

25. Liu M. G., Yan H. L., Wen G. Y., "Electrohydraulic Effect and Applications". Journal of Sichuan Institute of Technology, 8(3), 1989, pp. 188-193.

26. Zhang L., Deng Q. L., Zhou J. J., "Experimental Study of Shock Wave Effect of Electrical Discharge Under Water in Filth Cleaning". Journal of Dalian University of Technology, 38(2), 1998, pp.207-211.

27. OKun I. Z., "Plasma Parameters in a Pulsed Discharge in a Liquid". Soviet Physics -Technology Physics, 16(2), 1971, pp. 227-231.

28. Ushakov V. Y., et al., "Impulse Breakdown of Liquids". Berlin: Springer-Verlag, Germany, 2007, pp.37-45.

29. Zeng Z. H., "Research of large pulsed current test". Nanjing: Nanjing University of Science and Technology, China, 2006.

30. Liu Y. S., "Method of using P6015A detection head to measure DC high voltage". Electrotechnics Electric, 4(01), 2006, pp.42-44.

31. Zhang H. H., Chen Z. F., Lu X. P., "Measurement System in Underwater Wire Exploding Process". Journal of Test and Measurement Technology, 14(4), 2000, pp.221-225.

32. Martin E. A., "Experimental Investigation of a High-Energy Density". Journal of Applied Physics, 31(2), 1960, pp.255-259.

33. Wang J. F., Wang R. J., He S. A., "A measure of the fluid compressibility”. Physics, 10(03), 1981, pp.154-157. 